\title{
Albert Jesionek \\ auf der Spur der Zytomegalie-Krankheit
}

Peter Diosi

\section{Summary}

The first publications concerning the peculiar cellular structures that lateron became the hallmark of cytomegalic inclusion disease, originated in the early 20 th century from German institutes. These early reports got into oblivion even before their significance and implications could be fully realized. The purpose of this study is to reconsider the contributions of Jesionek and other authors to the discovery of the "protozoa-like structures", based upon their publications and in accordance with the state of knowledge at that time.

\section{Zusammenfassung}

Die ersten Veröffentlichungen über die eigentümliche Zellveränderung, die später zum Kennzeichen der Zytomegalie-Krankheit wurde, gingen Anfang des 20. Jahrhunderts aus deutschen Instituten hervor. Doch diese frühen Berichte waren, noch bevor ihre Bedeutung und Tragweite voll begriffen wurde, zum grössten Teil in Vergessenheit geraten. Daher dieser Versuch, die Beiträge Jesioneks und anderer Autoren zur Entdeckung der «protozoenartigen Gebilde» anhand ihrer Veröffentlichungen und gemäss der damaligen Kenntnise neu auszuwerten.

\section{Einführung}

Als Hugo Ribbert (1855-1920) 1880 in Bonn für Pathologie habilitiert hatte, interessierte er sich hauptsächlich für die Nierenpathologie, und zwar neben

Dr. Peter Diosi, Alteburger Strasse 298, D-50968 Köln 
der Entstehung der Albuminurie und Harnzylinder besonders für die Regenerationsvorgänge und die kompensatorische Hypertrophie der Nieren.

Rosenstein ${ }^{1}$ hatte 1871 noch behauptet, dass bei der komplementären Hypertrophie einer Niere - infolge erworbenen Schadens der anderen keine Vergrösserung der Glomeruli und der gewundenen Rindenkanälchen stattfindet. Er führte die Hypertrophie eher auf eine Zunahme des Gewichtes durch den vermehrten Gehalt des Organes an Blut, Lymph- und Harnbestandteilen und auf die durch gesteigerte Ernährung bewirkte grössere Dichte der einzelnen Elemente zurück. Perl ${ }^{2}$ aus Virchows Berliner pathologischem Institut konnte dagegen schon im darauffolgenden Jahr an 9 Fällen von einseitiger Nierenvergrösserung bei Hydronephrose zeigen, dass die Epithelien der gewundenen Harnkanälchen an Volumen erheblich zugenommen hatten. Doch für Ribbert war damit noch nicht entschieden, ob die Vergrösserung der Nieren auf Vermehrung (nach Virchows Terminologie Hyperplasie) oder auf Vergrösserung (Hypertrophie) der einzelnen Bestandteile beruht, und ob nur die gewundenen Harnkanälchen an Grösse zunehmen oder auch die Glomeruli und das Zwischengewebe daran beteiligt sind.

\section{Die kompensatorische Nierenhypertrophie}

Seine ersten Beobachtungen über die kompensatorische Nierenhypertrophie hatte Ribbert ${ }^{3}$ im Jahre 1881 in der «Niederrheinischen Gesellschaft für Natur- und Heilkunde» in Bonn, im Anschluss an eine Mitteilung seines Lehrers Köster ${ }^{4}$, vorgestellt. Sein Vortrag lief auf die erhebliche Volumenzunahme des Epithels der Harnkanälchen hinaus.

Er berichtete, dass bei fleckweiser interstitieller Nephritis eines totgeborenen luetischen Kindes in den Nieren inmitten entzündlich veränderter Abschnitte die Harnkanälchen völlig verschwunden waren, dagegen in der nächsten Umgebung derselben, wohl zur Ausfüllung der freigewordenen Räume, «eine sehr hübsche kompensatorische Hypertrophie» zeigten. Diese

1 S. Rosenstein, «Ueber complementäre Hypertrophie der Nieren.» Arch. path. Anat. Physiol. klin. Med. (Berlin), LIII/2-3, 141-155 (März 1871).

2 Leopold Perl, «Anatomische Studien über compensatorische Nierenhypertrophie.» Arch. path. Anat. Physiol. klin. Med. (Berlin), LVI/3, 305-315 (1872), S. 313-314.

3 Hugo Ribbert, «Partielle-compensatorische Hypertrophie der Harnkanälchenepithelien bei fleckweiser interstitieller Nephritis eines totgeborenen luetischen Kindes.» Sitzungsberichte der Niederrheinischen Gesellschaft für Natur- und Heilkunde (Bonn), 27. Juni 1881.

4 Karl Köster, «Beobachtungen bei partieller Hypertrophie interstitiell erkrankter Nieren.» Sitzungsberichte der Niederrheinischen Gesellschaft für Natur- und Heilkunde (Bonn), 27. Juni 1881. 
bestand darin, dass die an der Innenfläche der Harnkanälchen zwischen den normal grossen Epithelien entstandenen Lücken durch kolossal vergrösserte Zellen, deren Hauptmasse über die normalen Epithelien hinaus in das Harnkanälchenlumen hinausragte, ausgefüllt waren. «Ihr Querschnitt übertraf den normaler Zellen um das Zehnfache und auch der Kern war erheblich vergrössert und schien gegen das Protoplasma durch eine helle Zone scharf abgegrenzt zu sein.»

Dieser Befund hatte Ribbert ${ }^{5}$ dazu angeregt, die Frage der kompensatorischen Nierenhypertrophie an 2 jungen Hunden und 4 Kaninchen experimentell zu überprüfen. Doch im darüber 1882 veröffentlichten Bericht konnte er das Vorkommen ähnlicher Zellen bei den Versuchstieren nicht bestätigen.

\section{Umwandlung der Harnkanälchenepithelien}

Ribbert war vermutlich der erste Forscher der die grossen Zellen an der Innenfläche der Harnkanälchen bemerkt hatte. Fest steht, dass er diese Gebilde 1881 als kompensatorische, hypertrophische Veränderungen, also jedenfalls als eine Umwandlung der Organzellen, aufgefasst hatte. Diese Auslegung ergab sich daraus, dass er die Volumenzunahme der Zellen an der Stelle und nur dort angetroffen hatte, wo er solche aufgrund von Perls vorangegangenen Beobachtungen erwartet und vielleicht sogar gesucht hatte, nämlich in den Epithelien der Harnkanälchen.

Doch in seinen monochromen, mit Pikrokarmin gefärbten Präparaten konnte Ribbert damals die Anwesenheit eines voluminösen Einschlusskörpers in den Zellkern nicht wahrnehmen; er hielt diesen für den Zellkern, der jedoch homogen und matt gefärbt und gegen das Protoplasma durch eine helle Zone scharf abgegrenzt zu sein schien. Beeinträchtigt durch sein Interesse an der kompensatorischen Nierenhypertrophie hatte er die Möglichkeit eines Zusammenhanges zwischen den Zellgebilden und der Lues gar nicht in Betracht gezogen.

Erst einige Jahre später, als er während der Suche etwaiger entwicklungsgeschichtlicher Störungen die Gebilde in den Gängen der Kopfspeicheldrüsen zweier nichtluetischer Kinder entdeckt hatte, erkannte Ribbert ${ }^{6}$, dass

5 Hugo Ribbert, «Ueber compensatorische Hypertrophie der Nieren.» Arch. path. Anat. Physiol. klin. Med. (Berlin), LXXXVIII/1,11-27 (5. April 1882).

6 Hugo Ribbert, «Ueber protozoenartige Zellen in der Niere eines syphilitischen Neugeborenen und in der Parotis von Kindern.» Cbl. allg. Path. path. Anat. (Jena), XV/23, 945-948 (15. Dezember 1904). 


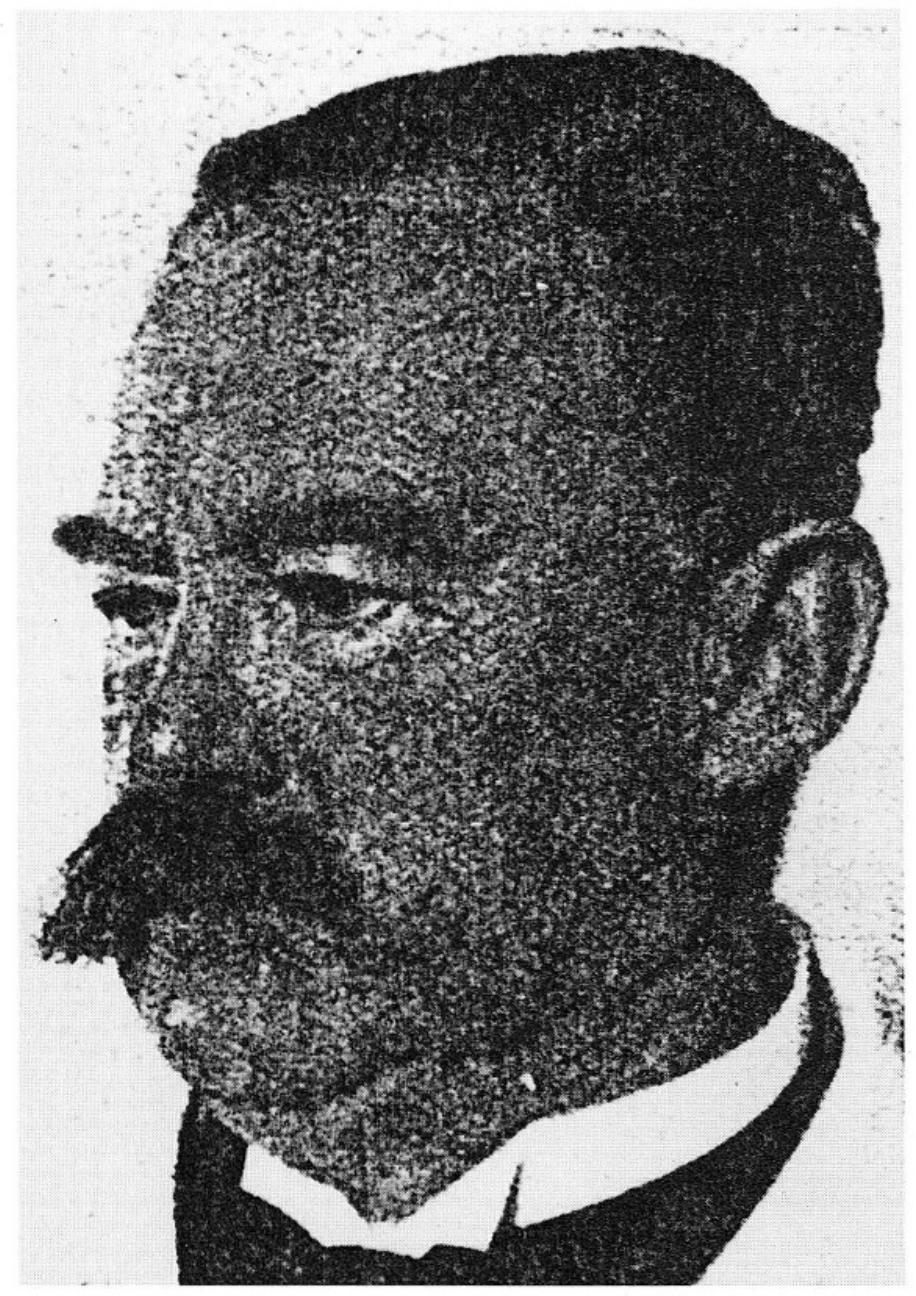

Abb. 1: Albert Jesionek (geb. 9.1.1870 zu Lindau in Bayern, $†$ 8. 12.1935 in Giessen.)

die eigentümlichen Elemente weder der kompensatorischen Nierenhypertrophie noch der Lues zugeschrieben werden konnten. Da ihm aber die Bedeutung der Gebilde nicht ausreichend klar werden wollte, hatte er seine Präparate vorerst beiseite gelegt.

\section{Die Entdeckung der Kerneinschlüsse}

Die Entdeckung der zytomegalen Kernkörper in menschlichen Gewebszellen wird auf einen Bericht von Jesionek und Kiolemenoglou ${ }^{7}$ in der am 25. Oktober 1904 erschienenen Ausgabe der «Münchener medizinische Wochenschrift» zurückgeführt.

7 Albert Jesionek und Basilius Kiolemenoglou, «Ueber einen Befund von protozoënartigen Gebilden in den Organen eines hereditär-luetischen Fötus.» Münch. med. Wschr., 51/43, 1905-1907 (25. Oktober 1904). 
Albert Jesionek (Abb. 1) war zuerst als Assistent, dann nach seiner 1901 erfolgten Habilitation für Dermatologie und Syphilidologie als Oberarzt an Posselts Münchener dermatologischen Klinik tätig. Im Sommer 1904 hatte er dort mit seinem Mitarbeiter Basilius Kiolemenoglou in den Organen einer 8monatigen, die spezifischen Eigentümlichkeiten der luetischen Erkrankung aufweisenden Totgeburt, eigenartige grosse Zellen entdeckt, welche sich von den bekannten Riesenzellen (Langhans-Zellen, Sternberg-Zellen usw.) durch die Anwesenheit eines eosinophilen Einschlusskörpers im Zellkern und mehrerer kleinerer Einschlüsse im Zelleib deutlich unterschieden. Die Plasmaeinschlüsse färbten sich noch dazu - im Gegensatz zu den damals bekannten, sich mit sauren Farbstoffen rotfärbenden Guarnerikörperchen bei Pocken oder Negrikörperchen im Spätstadium der Tollwut - mit basischen Farbstoffen dunkelblau an.

\section{Die Beschaffenheit der protozoenartigen Gebilde}

Jesionek berichtete, dass die Gebilde stark gequollenen Zellen glichen und die Grösse normaler Epithelzellen um ein 3-4faches übertrafen (Abb. 2, links oben).

Der Kern war meistens polständig und bestand aus einem scharf hervortretenden, mit Eosin rötlich gefärbten zentralen Kernkörper, umgeben von einem hellen Hof, der vom Zelleib durch die Kernmembran wie von einer Schale abgegrenzt zu sein schien. An der Wand der Umgrenzung, im hellen Hof, fielen ihm mehrere kreisrunde mit Hämatoxylin dunkelblau gefärbte Körperchen auf. Die Beschaffenheit des Zelleibes hatte Jesionek als schwammig beschrieben, mit einer an der dem Kern gegenüberliegenden Seite deutlichen Anhäufung blau gefärbter Körner, die besonders distal angesammelt waren und medial die schwammartige Grundsubstanz deutlich hervortreten liessen.

Einige abweichende Erscheinungsbilder liessen sich unschwer aus der verschiedenen Höhe der Schnittführung erklären, doch auf andere Verschiedenheiten hinsichtlich der Beschaffenheit der Gebilde versprach Jesionek anhand von Abbildungen an anderer Stelle noch zurückzukommen.

\section{Das Verbreitungsmuster}

Die eigentümlichen Gebilde waren Jesionek ausser in den Nieren auch in den Lungen und in der Leber begegnet. In den Nieren hatte er sie nicht in 


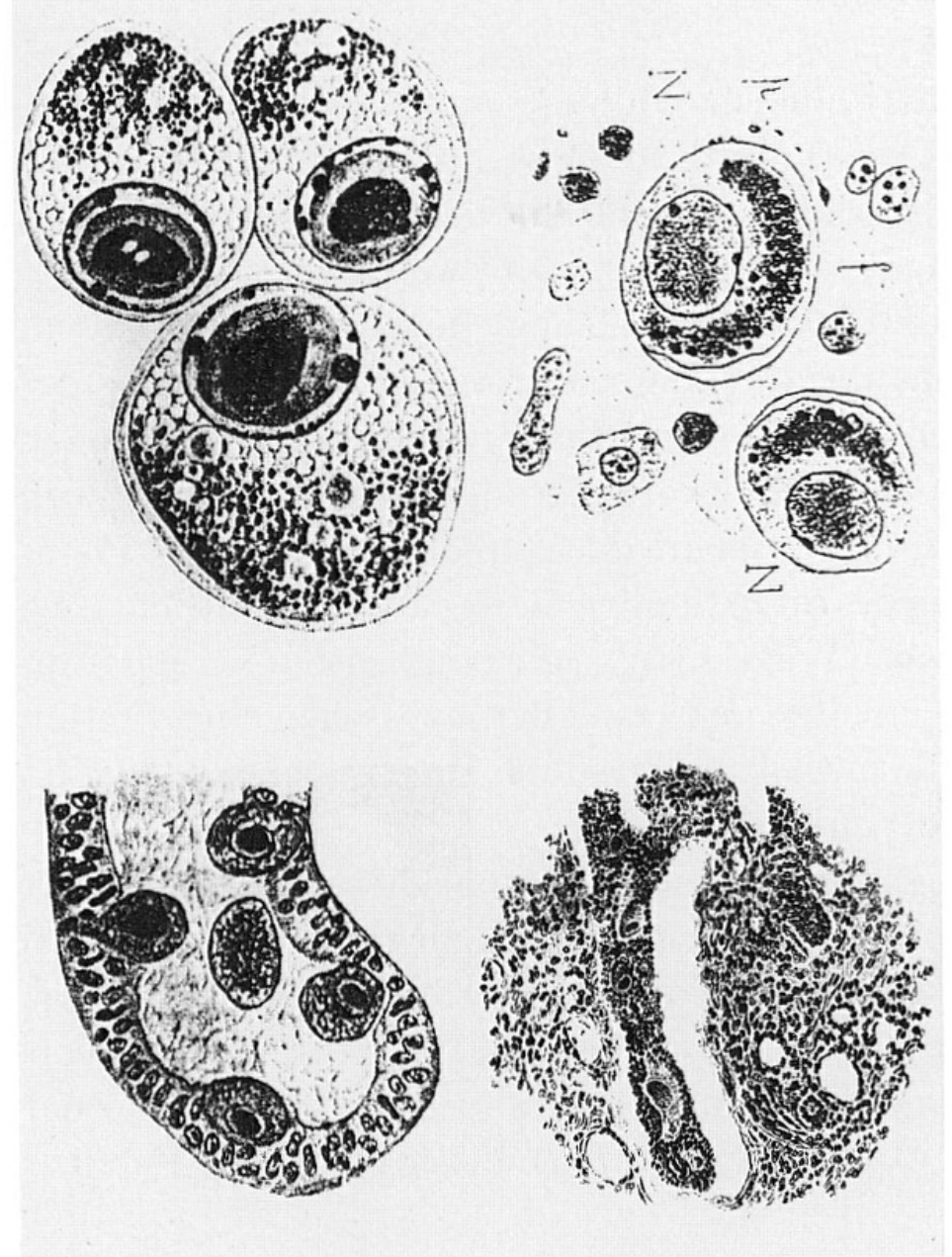

Abb. 2:

Links oben: Felix v. Cubes halbschematische Zeichnung von «protozoenartigen Zellgebilde» (nach Jesioneks Originalpräparat).

Rechts oben: Anglas' Skizze von den im Harnkanälchen eines mazerierten Föten begegneten «Gigantischen» Zellen.

Links unten: Ribberts Darstellung von «protozoenartigen Zellen» im Epithelverband eines Speichelganges.

Rechts unten: Tietzes Darstellung der als Amöben betrachteten Riesenzellen in einem der Drüsenschläuche der Parotis.

den Harnkanälchenepithelien, sondern inmitten des interstitiellen Gewebes regellos, in Gruppen vereint oder einzeln zerstreut angetroffen. In den Lungen und in der Leber dagegen hatte er verhältnismässig wenige Gebilde und fast ausschliesslich als einzeln gelagerte Elemente gefunden.

Das auf die Nieren, Lungen und die Leber verstreute Vorkommen der protozoenartigen Gebilde hat sich später als eines ihrer häufigsten Verbreitungsmuster erwiesen. 


\section{Die Ursprünglichkeit der Gebilde}

Frühere Beobachtungen über ähnliche Gebilde waren Jesionek nicht bekannt; von Ribberts Arbeit wusste er nicht. Dessen Vortrag hatte doch vor Jahrzehnten, noch im 19. Jahrhundert, in Bonn am Niederrhein im Kreise von Pathologen stattgefunden und wurde seitdem nicht weiter erwähnt.

Doch selbst wenn ihm Ribberts Vortrag bekannt gewesen wäre, erscheint es fraglich, ob Jesionek, aus der die kompensatorische Hypertrophie der tubulären Nierenepithelien schildernden Beschreibung hätte erkennen können, dass es sich da um dieselbe Zellveränderung handelte. Er hatte doch die seltsamen Gebilde nicht wie Ribbert im Epithelialverband der Harnkanälchen, sondern inmitten des interstitiellen Gewebes «... gerade dort, wo die Äusserungen des luetischen Prozesses (...) die deutlichsten Veränderungen geschaffen haben», angetroffen und Dank der inzwischen eingeführten Differentialfärbung auch die auffälligen eosinophilen Einschlusskörper im vergrösserten Zellkern sofort entdeckt.

Als Jesionek der Einzigartigkeit und der möglichen Bedeutung der Gebilde gewahr wurde, entschied er sich unverzüglich, einen vorläufigen Bericht über den sonderbaren Zellbefund zu veröffentlichen. Die Vorgeschichte des Falles und die makroskopischen und mikroskopischen Veränderungen in den Organen betreffend, verwies er dabei auf eine in der Münchener medizinischen Wochenschrift demnächst erscheinende Publikation.

\section{Was hatte Jesionek zur Eile veranlasst?}

Burmester $^{8}$ aus dem Tübingener pathologischen Institut hat später die Tatsache, dass die protozoenartigen Zellen anfangs in luetischen Föten gesichtet wurden, darauf zurückgeführt, dass vor der Entdeckung der Treponema pallidum durch Schaudinn und Hoffmann im Jahre 1905 die Gewebe luetischer Kinder mit besonderer Sorgfalt nach dem vermuteten Erreger der Syphilis überprüft wurden.

Möglicherweise hatte Jesionek ihre Beziehung zur Lues im Auge, als er die im Sommer 1904 entdeckten Elemente schon in der Oktober-Ausgabe desselben Jahrgangs der Münchener medizinischen Wochenschrift, und zwar gesondert, noch vor der Veröffentlichung der Krankengeschichte und der pathologischen Befunde des Falles, bekannt gab.

8 Friedrich Burmester, «Das Speicheldrüsenvirus des Menschen (Cytomegalia infantum).» Virchows Arch., 317/1-2, 165-189 (5. Oktober 1949), S. 167. 
Dabei muss hervorgehoben werden, dass es sich laut Jesionek um einen seltenen Fall gehandelt haben soll, wo die Mutter in keiner Weise mit einem der damals gebräuchlichen antiluetischen Mittel behandelt worden war und die Organe des Fötus die spezifischen Eigentümlichkeiten der luetischen Erkrankung in einer Vollkommenheit und Reinheit aufwiesen, wie das sonst nur selten zur Beobachtung kam.

Auch als er später die Vorgeschichte und die pathologischen Befunde des Falles veröffentlicht hat, erinnerte Jesionek ${ }^{9}$ der Vollständigkeit halber erneut daran, dass sich in den Organen des Fötus protozoenartige Zellgebilde fanden.

\section{Die mögliche Beziehung zur Syphilis}

Die Gebilde stellten zweifelsohne eine überraschende Neuigkeit dar und warfen die Frage auf, ob diese Veränderungen nicht dem Erreger der Syphilis zuzuschreiben seien, ja dieser selbst darin zu suchen sei. Jesionek hatte sie doch in einem Fall von angeborener Syphilis inmitten luetischer Veränderungen entdeckt.

Es scheint, dass Jesionek die kausale Beziehung der Gebilde zur Lues anfangs zwar erwogen, aber davon gleich Abstand genommen hatte, da der doch gewiss auffallende Befund bis dahin im Laufe von zahllosen Untersuchungen luetischer Geweben niemals erhoben worden war.

Nebenbei sei erwähnt, dass Kiolemenoglou ${ }^{10} 1905$ die kausale Rolle der von Schaudinn entdeckten Spirochäten bezweifelt hatte.

\section{Schwierigkeiten bei der Deutung der Gebilde}

Die Deutung der eigentümlichen Elemente hatte Jesionek nach eigener Angabe auch zur Zeit der Abfassung seines Berichtes noch grosse Schwierigkeiten bereitet. Er war sich nicht sicher darüber, ob sie körpereigene Zellen oder in die Gewebe eingedrungene Schmarotzer seien.

Waren sie Gewebszellen, so stellte sich die Frage, ob es sich um rückständige embryonale Zellen oder differenzierte Körperzellen, und zwar um zersprengte Keime oder fixe Gewebszellen handelte, und wodurch und auf

9 Albert Jesionek, «Ein Beitrag zur Lehre von der Vererbung der Syphilis.» Münch. med. Wschr., LI/49, 2172-2175 (6. Dezember 1904) und LI/50, 2231-2235 (13. Dezember 1904).

10 Basilius Kiolemenoglou und Felix v. Cube, «Spirochaete pallida (Schaudinn) und Syphilis.» Münch. med. Wschr., LII/27, 1275-1276 (4. Juli 1905). 
welche Weise es zu diesen eigentümlichen Veränderungen der Zellen kam. Waren sie jedoch Parasiten, so mussten sie identifiziert und in die entsprechende Gattung eingeordnet werden.

\section{Heterotope Zellen?}

Ove-Hamburger und andere Forscher hatten in der fötalen Niere im Zusammenhang mit entwicklungsgeschichtlichen Vorgängen schon früher das Vorkommen eigenartiger Zellformen betont. Doch Jesionek schienen die protozoenartigen Zellen den in der Literatur zugänglichen Schilderungen nicht zu entsprechen.

Die Möglichkeit, dass es sich um versprengte Keime handelte, konnte Jesionek im Vergleich mit den herangezogenen Zellen anderer Organe desselben Fötus durch den Mangel jeglicher Ähnlichkeit hinsichtlich Form und Grösse mit an Sicherheit grenzender Wahrscheinlichkeit ausschliessen.

\section{Mazerationserscheinungen?}

Da die Autolyse der Gewebe nur geringfügig war und die seltsame Veränderung nur wenige, zerstreut liegende Zellen betraf, konnte es sich Jesionek nicht vorstellen, dass die Gebilde auf Mazerationseinflüsse zurückzuführen seien. Ähnliche, verdächtig erscheinende Zellen, welche ihm bei einem zweiten luetischen Fötus aufgefallen waren, getraute er sich eben wegen der in diesem Falle beträchtlichen Mazerationserscheinungen nicht als protozoenähnliche Strukturen zu identifizieren.

Hier sei jedoch erwähnt, dass im Jahre 1909 Anglas $^{11}$ die in den Harnkanälchen eines mazerierten Fötus beobachteten ähnlichen Gebilde unmittelbar für autolytisch veränderte Körperzellen hielt ${ }^{12}$.

\section{Degenerierte Körperzellen?}

Ebenso unwahrscheinlich erschien es Jesionek, dass Parenchymzellen dreier anatomisch so verschiedener Organe wie die Nieren, Lungen und die Leber

11 Jules Anglas, «De l'histolyse et de l'autolyse des tissues fœtaux macérés. Suite et fin.» J. Anat. Physiol. (Paris), XLV/5, 400-432 (Septembre-Octobre 1909), S. 403-404.

12 Peter Diosi, «Les observations du Docteur Anglas sur la présence de cellules cytomégaliques dans les tissus fœtaux macérés.» Arch. Anat. Cytol. path. (Paris), 44/4, 188-192 (Juillet 1996). 
durch Degeneration in ein- und derselben Weise verändert worden bzw. dass eine unter Degenerationseinfluss entstandene Umwandlung auf wenige zerstreute Organzellen beschränkt sei. Es fehlten ausserdem jegliche Übergangsformen von den normalen Körperzellen zu den seltsamen Gebilden, welche als Phasen der Umwandlung hätten betrachtet werden können.

\section{Erwägung der parasitären Natur}

Es wurde dagegen schon lange vermutet, dass in der Ätiologie der angeborenen Lues, ähnlich wie bei Malaria und Amöbenruhr, Protozoen eine Rolle spielen könnten. Da ihm die Gebilde mitten in den Geweben als «fremde Eindringlinge» erschienen, erwog auch Jesionek die Möglichkeit ihrer parasitären Natur und konsultierte diesbezüglich den illustren Protistologen Richard Hertwig. Dieser hat ihm daraufhin bestätigt, dass die fraglichen Gebilde Protozoen, und zwar eine Art Gregarinen, seien.

Es gibt mehrere Hinweise dafür, dass Jesionek von Hertwigs Auslegung nicht restlos überzeugt war. In diesem Sinne versteht sich erstens seine Aussage, wonach die Deutung der Gebilde ihm auch zur Zeit der Abfassung seines Berichtes noch grosse Schwierigkeiten bereitete. Darauf weist auch die Tatsache hin, dass er in seinem Bericht, wie auch in später über das pathologische Bild veröffentlichten Mitteilungen ${ }^{13}$, statt von Gregarinen weiterhin von «protozoenartigen Zellgebilden» sprach, und das beweisen schliesslich seine Bemerkungen, wonach die Mutter des Fötus keinerlei Krankheitserscheinungen aufwies welche auch nur entfernt eine Infektion mit Blut- oder Darmparasiten annehmen liesse bzw. dass Gregarineninfektionen beim Menschen bis dahin nicht bekannt geworden waren.

Mit der suggestiven Bezeichnung «protozoenartige Gebilde» hingegen, gelang es Jesionek diesen Zellen eine genaue Identität zu verleihen und so die Bezugnahme auf diese zu erleichtern.

\section{Ribberts alte Präparate}

Ribbert ${ }^{14}$, der mittlerweile 1892 in Zürich Ordinarius geworden war, dann 1900 eine Berufung nach Marburg angenommen hatte und schliesslich 1903 nach Göttingen übersiedelt war, fiel beim Lesen von Jesioneks Bericht 13 Jesionek (No. 8), S. 2233.

14 Ribbert (No. 5), S. 946. 
wieder ein, dass er vor etwa 20 Jahren als Kösters Assistent in Bonn, in den Nieren einer syphilitischen Totgeburt und einige Jahre später in den Kopfspeicheldrüsen zweier nichtsyphilitischer Kinder ähnliche Gebilde schon beobachtet hatte. Jesioneks Bericht veranlasste ihn nun, auch seine Beobachtungen umgehend zu veröffentlichen.

Bei der erneuten Untersuchung seiner vor zwei Jahrzehnten beiseite gelegten und unterdessen etwas verschwommenen Präparate erschien ihm jetzt, dass sich in den Kernen der vergrösserten Zellen tatsächlich ein zentraler Einschlusskörper befand, der aber nicht in allen Fällen sichtbar war.

Sein Assistent, Löwenstein ${ }^{15}$, hatte darauf aufmerksam gemacht, dass Jesionek und Ribbert den Ausdruck «Kernkörper» nicht in gleichem Sinne angewandt hatten. Während Jesionek den gesamten mit Eosin rötlichgefärbten und vom hellen Hof umgebenen Kerninhalt als Kernkörper bezeichnet hatte, betrachtete Ribbert diese Struktur weiterhin als den Zellkern, der sich von der Membran zurückgezogen zu haben schien, und bezeichnete als Kernkörper lediglich den mittleren, manchmal etwas dunkler erscheinenden Teil des Einschlusses.

\section{Ribberts revidierte Auslegung}

Da nur die von den zwei nicht-syphilitischen Kindern stammenden Präparate im gutem Zustand erhalten waren, befasste sich Ribbert diesmal hauptsächlich mit dem auf die Kopfspeicheldrüsen begrenzten Vorkommen der Gebilde.

Hatte er ursprünglich aufgrund der strengen Einordnung der vergrösserten Zellen in den Epithelialverband der Harnkanälchen noch deren Abstammung von Epithelien angenommen, so erinnerten ihn die Gebilde jetzt eher an dem Epithelverband der Speichelgänge anliegende oder sich zwischen die Zellen «hineindrängende» Protozoen (Abb. 2, rechts oben).

\section{Verkehrte Auswirkung}

Ribbert soll den Wert seiner Mitteilung hauptsächlich darin gesehen haben, dass sie «den Eindruck der durch Jesionek und Kiolemenoglou beigebrachten Tatsachen verstärkend, zu weiteren Untersuchungen auffordert». Doch ausser Löwenstein war jahrelang niemand seiner Aufforderung gefolgt. Statt

15 Carl Löwenstein, «Ueber protozoënartige Gebilde in den Organen von Kindern. Vorläufige Mitteilung.» Cbl. allg. Path. path. Anat. (Jena), XVIII/13, 513-518 (15. Juli 1907), S. 513-514. 
dessen brachte seine Mitteilung Jesionek von der angekündigten Veröffentlichung weiterer Beobachtungen kurzerhand ab. Mit dem Hinweis auf seine früheren Befunde hatte Ribbert nämlich nicht nur die Priorität der beiden Dermatologen in Frage gestellt, sondern auch die Vormundschaft über die weitere Erforschung dieser Strukturen übernommen. Die Entdeckung der grossen Zellen in den Speicheldrüsen nichtluetischer Kinder hatte ja deutlich gezeigt, dass die Gebilde nicht zum Bereich der Syphilidologie gehörten. Die meisten Pathologen würdigten daher Ribbert als den Entdecker und betrachteten die seltsamen Gebilde als Teil ihres Fachbereiches.

Anfang 1906 wurde Jesionek dann zum ausserordentlichen Professor der Dermatologie befördert und gleich darauf, im Mai 1906, auf den neu geschaffenen Lehrstuhl der Hautkrankheiten an der Universität Giessen berufen. Dort hatte er 1913 die erste deutsche Lupusheilstätte ins Leben gerufen und sich im weiteren der Licht-, Tuberkulin- und Diätbehandlung der Hauttuberkulose gewidmet. In keinem der Nachrufe zu seinem am 8. Dezember 1935 erfolgten Tode wurde die Entdeckung der protozoenartigen Gebilde erwähnt.

\section{Besiedlung der Speicheldrüsen}

Nachdem Ribbert ${ }^{16} 1905$ als Kösters Nachfolger nach Bonn zurückgekehrt war, erschien ihm die Frage der protozoenartigen Gebilde bei seinen sonstigen wichtigen Untersuchungen zu unbedeutend, um sich weiter damit zu befassen. Er konzentrierte sich daher auf die Vervollständigung seiner Geschwulstlehre und überliess seinen Assistenten die Aufgabe «auf die pathologischen Wachstumsvorgänge an der kindlichen Parotis zu achten».

Daraufhin hat Löwenstein ${ }^{17} 1907$ bei einer Reihe von 30 Kleinkindern die Speicheldrüsen mituntersucht, und traf in 4 Fällen ausgerechnet dort und nur in den Speicheldrüsen auf protozoenartige Zellen. Diese Einschränkung der Gebilde auf die Speicheldrüsen veranlasste ihn zur Annahme, dass die Parotis der Haupsitz dieser Elemente sei.

Als sich später die Speicheldrüsen auch bei den Meerschweinchen als Standort ähnlicher Gebilde erwiesen haben, etablierte sich in der angelsächsischen Literatur allmählich der Begriff «salivary gland disease».

16 Hugo Ribbert, «Karl Köster (1843-1904). (Necrolog)». Cbl. allg. Path. path. Anat. (Jena), XV/23, 977-981 (1904).

17 Löwenstein (No. 15), S. 514. 


\section{Spätere Entdeckungen ähnlicher Gebilde}

Die routinemässige mikroskopische Untersuchung der pathologischen Gewebsveränderungen erlaubte im frühen 20. Jahrhundert auch anderen Beobachtern die Entdeckung ähnlicher Elemente (Abb. 2, unten, links und rechts). So wurden solche im Ablauf von etwa 30 Jahren in mehreren Ländern unabhängig voneinander von mehreren Autoren entdeckt, namentlich von Tietze ${ }^{18}$ in Deutschland, Anglas ${ }^{19}$ in Frankreich, Hesselberg ${ }^{20}$ (zit. von Pettavel $^{21}$ ) in der Schweiz, Pisano ${ }^{22}$ und Castelli ${ }^{23}$ in Italien, Wanstrom ${ }^{24}$, Wilson und DuBois ${ }^{25}$ in den Vereinigten Staaten und Malowitschko und Pupenko ${ }^{26}$ in der Ukraine. Offenbar waren diesen Autoren die vorangegangenen Beobachtungen über die eigentümlichen Gebilde vollkommen unbekannt.

\section{Unterschiedliche Beurteilung der Gebilde}

Alle Beobachter waren verwundert über den eigentümlichen Zellbefund, doch ihr Verhalten angesichts der für die einen unbedeutenden, für die anderen undeutbaren Gebilde war, je nach ihrem Temperament, sehr unterschiedlich.

Ribbert assoziierte die kolossal vergrösserten Zellen anfangs mit der kompensatorischen Nierenhypertrophie, weil diese seiner durch Perls Beschreibung erweckten Erwartungen zu entsprechen schienen. Hesselberg, aus dem Langhans'schen pathologischen Institut in Bern, hatte dagegen die in der

Schilddrüse eines an Purpura verstorbenen Kindes entdeckten grossen

18 Alexander Tietze, «Ein Protozoenbefund in einer erkrankten Parotis.» Mitt. Grenzgeb. Med. Chir., 14/3, 303-310 (1905).

19 Anglas (No. 8).

20 Cora Hesselberg, «Die menschliche Schilddrüse in der fötalen Periode und in den ersten 6 Lebensmonaten (Inaugural Dissertation, Jena).» Frankfurt. Z. Pathol., 5, 322-350 (1910).

21 Charles A. Pettavel, «Über eigentümliche herdförmige Degenerationen der ThyreoideaEpithelien bei Purpura eines Neonatus.» Virchows Arch. path. Anat., 206/1, 1-10 (13. Oktober 1911).

22 G. Pisano, «Su di un reperto istologico raro in feto scleromatoso.» Gazz. Osp. Clin., XXXI/24, 249-250 (24 febbraio 1910).

23 Giorgio Castelli, «Anomalia istologica in rene di feto umano. Uova primordiali aberranti?» La Ginecologia (Firenze), VII/9, 257-276 (15 maggio 1910).

24 Ruth C. Wanstrom, «Erythroleukoblastosis in the Newborn.» Am. J. Path., 9/2, 623-635 (March 1933).

25 James R. Wilson and R. O. DuBois, «Report of a Fatal Case of Keratomalacia in an Infant with Post Mortem Examination.» Am. J. Dis. Child., 26/5, 431-446 (November 1923).

26 Eugenia Malowitschko und I. G. Pupenko, «Ein Fall von Amöbenansteckung der menschlichen Unterkieferdrüse.» Arch. Schiffs. Tropenhyg., 38/1, 28-31 (Januar 1934). 
Zellen nicht ins Konzept gepasst. Obwohl diese sofort den Eindruck eines seltsamen Befundes erweckten, fand er für sie im Bereich seiner Untersuchungen keine Verwendung und überliess die weitere Bearbeitung des Falles Pettavel ${ }^{27}$. Andere Forscher hatten ihre Präparate beiseite gelegt, weil ihnen die Bedeutung der Gebilde nicht ausreichend klar werden wollte. So fand z.B. Tietze ${ }^{28}$ aus dem Breslauer Augustahospital in den Drüsengängen einer von der Parotis eines Säuglings entfernten Geschwulst eigentümliche grosse Zellen, die er für aus der Mundhöhle eingewanderte Amöben hielt. Er verzögerte jedoch 7 Jahre lang die Veröffentlichung des Befundes, angeblich wegen anderweitiger Beschäftigungen ${ }^{29}$. Aschoff ${ }^{30}$ beschränkte sich in der 1921er Ausgabe seines Lehrbuches der pathologischen Anatomie auf die Inserierung einer Abbildung der in den Nieren eines syphilitischen Kindes entdeckten grossen Zellen, ohne über sie im Text nähere Angaben zu machen. Laut Wagner ${ }^{31}$ aus Aschoffs Freiburger pathologischem Institut waren die eigentümlichen Zellen von den meisten Untersuchern als Nebenbefund aufgefasst, dem im allgemeinen keine primär pathogene Bedeutung zukommt. Er selbst hatte jegliche Bedeutung der Gebilde abgestritten und stellte sie als mikroskopische Kuriositäten hin.

Goodpasture $^{32}$, dem es 1921 gelungen war ihre Abstammung von Gewebszellen zu beweisen, bezeichnete diese Umwandlung als «Zytomegalie» (Cytomegalia), um damit die betroffenen Zellen von Protozoen, aber auch von den übrigen Riesenzellen deutlich zu unterscheiden.

\section{Die Vorahnung einer eigenständigen Krankheit}

Pisano ${ }^{33}$ sowie Perrando ${ }^{34}$ hatten noch angenommen, dass bei der Entstehung der eigentümlichen Gebilde die Syphilis eine Rolle spielt, und Müller ${ }^{35}$

27 Pettavel (No. 19), S. 1.

28 Tietze (No. 18), S. 308-309.

29 Peter Diosi, «Tietze und die Zytomegalie-Erkrankung der Speicheldrüsen.» Gesnerus, 53/1-2, 87-99 (Juni 1996).

30 Ludwig Aschoff, Lehrbuch der pathologischen Anatomie, 5. Aufl., Ed. Fischer, Jena, 1921, S. 500.

31 Hans Wagner, «Zur Kenntnis der «protozoenartigen Zellen» in den Organen von Kindern.» Ziegler, Beitr. path. Anat., LXXXV/1,145-161 (1930), S. 163.

32 Ernest W. Goodpasture and Fritz B. Talbot, «Concerning the Nature of «Protozoan-Like» Cells in Certain Lesions of Infancy.» Amer. J. Dis. Child., 21/5, 415-425 (May 1921).

33 Pisano (No. 25).

34 G. G. Perrando, «Per l'interpretazione di taluni elementi eccezionali riscontrati in visceri di neonati.» Pathologica, IV/86, 310-312 (1 giugno 1912).

35 I. Müller, «Ueber die protozoenartigen Gebilde in den Harnkanälchenepithelien Neugeborener.» Virchows Arch. path. Anat., 238/3, 481-494 (1922). 
aus Lubarschs Berliner pathologischem Institut hatte die Veränderungen schlechtweg als syphilitisch aufgefasst.

Jakubowicz $^{36}$ aus v. Meyenburgs Züricher pathologischem Institut behauptete dagegen, dass bei keinem der bis 1930 veröffentlichten Fälle die luetische Infektion in einwandfreier Weise nachgewiesen werden konnte. Diese Tatsache veranlasste ihn, die Möglichkeit in Betracht zu ziehen, dass eine nicht näher bekannte, der Lues ähnliche, jedoch eigenständige Krankheit mit den protozoenartigen Zellen zusammen bestehen, ja sogar durch diese verursacht sein könnte.

\section{Die Abgrenzung gegen ähnliche Krankheiten}

Nach der Einführung der Wassermann-Reaktion wurde laut Vidari ${ }^{37}$ das Vorkommen von Ikterus, Ödemen und ausgedehnten Zellinfiltrationen in den seronegativen Fällen statt der Syphilis immer häufiger der hämolytischen Krankheit der Neugeborenen zugeschrieben. Gierke ${ }^{38}$ hatte nämlich um 1930 erkannt, dass diese auch bei der Lues oft begegneten Symptome in Wirklichkeit nicht Merkmale der Syphilis, sondern verschiedene Erscheinungen ein und derselben hämolytischen Krankheit der Neugeborenen darstellten. So kam allmählich der Gedanke auf, dass die Gebilde mit dieser Krankeit im Zusammenhang stünden.

Als aber die hämolytische Krankheit auf eine Rh-Blutgruppen-Inkompatibilität zurückgeführt wurde, gelang es Cappell und McFarlane ${ }^{39} \mathrm{zu}$ beweisen, dass in den von protozoenähnlichen Zellen gekennzeichneten Fällen keine Blutgruppen-Inkompatibilität bestand und die hämolytischen Erscheinungen möglicherweise infolge einer, durch die Ausstreuung des vermuteten Speicheldrüsenvirus verursachten, eigenständigen Krankheit auftraten.

36 Aba Jakubowicz, «Über protozoenartige Gebilde in Zellen verschiedener Organe des Menschen und ihr Zusammenhang mit angeborener Syphilis.»Virchows Arch. path. Anat., 276/2, 290-304 (6. März 1930).

37 Ettore Vidari, «Ein Fall von erythrämischer Myelose mit Befund von protozoenähnlichen Zellen.» Zbl. allg. Path. path. Anat. (Jena), 76/5, 161-166 (18. Dezember 1940).

38 Edgar O. C. von Gierke, «Über fetale Erythro-Leukoblastose.»Virchows Arch. path. Anat., 275, 330-347 (1930).

39 D. F. Cappell and Marjory N. McFarlane, «Inclusion Bodies (Protozoon-like Cells) in the Organs of Infants.» J. Path. Bact., LIX, 385-398 (1947), S. 393-396. 


\section{Die Vielfalt der Erscheinungsbilder}

Im Laufe der Zeit wurde das Vorkommen protozoenähnlicher Gebilde mit immer mehr Krankheiten in Verbindung gebracht. Tietze ${ }^{40}$ fand sie in einer dem Mikulicz-Syndrom ähnlichen Erkrankung der Parotis, Mouchet ${ }^{41}$ in Icterus neonatorum, McCordock und Smith ${ }^{42}$ in Keuchhusten, und andere Forscher entdeckten sie in einem Toxoplasmose-ähnlichen Syndrom ${ }^{43}$. Hartmann $^{44}$ aus Chiaris Wiener pathologischem Institut konnte 1948 noch mehrere mit den protozoenartigen Zellen in Zusammenhang gebrachte Grundkrankheiten und Symptomen-Komplexe auflisten (u.a. Leberzirrhose, Hydrops congenitus, angeborene Hämoblastose, Purpura neonatorum, Hydrocephalus bzw. Mikrocephalus congenitus usw.), doch ihre Beziehung zu mehreren Krankheiten erschien bald unwahrscheinlich.

Wyatt ${ }^{45}$ kam 1950 zur Schlussfolgerung, dass die Vielfalt der Krankheitserscheinungen durch die unterschiedlichen Verbreitungsmuster der Gebilde bestimmt sei und eigentlich verschiedene Erscheinungsbilder derselben Krankheit darstellt. Er bezeichnete diese als «Cytomegalic inclusion disease».

\section{Klärung der Ätiologie}

Mouchet ${ }^{46}$ gelangte schon 1911 zur Einsicht, dass es bei den protozoenartigen Gebilden nicht um in den Epithelialverband eindringende Protozoen, sondern um die Umbildung der von intrazellulären Parasiten befallenen Epithelien und deren spätere Abstossung handelte ${ }^{47}$.

40 Tietze (No. 18).

41 René Mouchet, «De la présence de protozoaires dans les organes des enfants. Contribution à l'étude de l'ictère des nouveau-nés» Arch. Méd. exp. (Paris), 23/2, 115-124 (Mars 1911), S. 121.

42 Howard A. McCordock and Margaret G. Smith, «Intranuclear Inclusions. Incidence and Possible Significance in Wooping Cough and in a Variety of Other Conditions.» Am. J. Dis. Child., 47, 771-779 (April 1934).

43 Albert B. Sabin and Harry A. Feldman, «Chorioretinopathy Associated with Other Incidence of Cerebral Damage in Childhood: Syndrome of Unknown Etiology Separable from Congenital Toxoplasmosis.» J. Pediat., 35/3, 296-309 (September 1949).

44 Gottfried Hartmann, «Über die 〈protozoenartigen Zellen〉 in den Organen Neugeborener.» Klin. Med. (Wien), 3/8, 281-293 (15. April 1948).

45 John P. Wyatt, J. Saxton, R. S. Lee and Henry Pinkerton, «Generalized Cytomegalic Inclusion Disease.» J. Pediat., 36/3, 271-294 (March 1950).

46 Mouchet (No. 41).

47 Peter Diosi, «Note sur la contribution du docteur René Mouchet à l'étude de l'ictère des nouveau-nés.» Rev. med. Liège., 51/4, 313-316 (1996). 
Als Lipschütz ${ }^{48} 1921$ auch in dem als Virusinfektion geltenden Herpes das Vorkommen ähnlicher Kerneinschlüsse nachweisen konnte, dämmerte es schon manchen Forschern, dass der von Mouchet vermutete intrazelluläre Parasit eigentlich ein unsichtbares Virus sei. Minder ${ }^{49}$ aus Walthards Berner pathologischem Institut gelang es sogar, die Elementarkörperchen innerhalb der befallenen Zellen elektronen-optisch sichtbar zu machen. Doch den endgültigen Beweis der Virusätiologie konnten Weller ${ }^{50}$ und andere Forscher durch die Isolierung des menschlichen Speicheldrüsenvirus (salivary gland virus, später Cytomegalovirus) erst 1956 erbringen.

Seifert $^{51}$ aus dem Leipziger pathologischen Institut und Oehme aus der Marburger Kinderklinik haben dann an der Schwelle des virologischen Zeitalters, die frühen Errungenschaften der Zytomegalieforschung abschliessend in einer Monographie zusammengefasst.

\section{Nachwort}

Die frühen Beobachter waren rein zufällig auf protozoenartige Zellen gestossen, doch das Antreffen der Gebilde durch mehrere Forscher unabhängig voneinander zeigt, dass es nach der Einführung der Differentialfärbung zwangsläufig zu dieser Entdeckung kommen musste.

Beachtet man weiter, dass jene Berichte, welche den eigentümlichen Zellbefund unbenannt liessen oder entschieden als Protozoenbefund vorstellten, seit langem vergessen in den Archiven ruhen, so kann Jesioneks Einfluss auf die spätere Entwicklung darauf zurückgeführt werden, dass er den Gebilden Individualität geschenkt hat, und damit das Interesse an dieser Zellveränderung über die Zeit der konzeptuellen Umwandlungen hinweg rettete.

Es dauerte jedoch ein halbes Jahrhundert bis die Beobachtungen über die begleitenden Symptomen-Komplexe schliesslich ein neues Krankheitsmuster ergaben, in welches sich die im Kontext der Syphilis und anderer Krankheiten als Störfaktor geltenden Gebilde nun als sinnvolle Erscheinungen hineinfügten, das eigene Bild der Zytomegalie-Krankheit.

48 Benjamin Lipschütz, «Untersuchungen über die Krankheiten der Herpesgruppe (Herpes zoster, Herpes genitalis, Herpes febrilis).» Arch. Dermat. Syph., 136/3, 428-482 (2. Dezember 1921).

49 W. H. Minder, «Die Ätiologie der Cytomegalia infantium.» Schweiz. med. Wschr., 83/49, 1180-1182 (1953).

50 Thomas H. Weller,I. C. Macauley,J. M. Craig and P. Wirth, «Isolation of Intranuclear Inclusion Producing Agents from Infants with Illnesses Resembling Cytomegalic Inclusion Disease.» Proc. Soc. exp. Biol. Med., 94/1, 4-12 (1957).

51 Gerhard Seifert und Johannes Oehme, «Pathologie und Klinik der Cytomegalie.» Ed. VEB Georg Thieme, Leipzig, 1957. 